\title{
Reducing frequent visits to emergency departments
}

Jane George ${ }^{1}$ and Lynne Briggs ${ }^{2}$

\begin{abstract}
BACKGROUND: The Health and Wellbeing Connection (HWC) pilot study was undertaken by Richmond Services in partnership with Pegasus Health, Partnership Health and the Canterbury District Health Board.

OBJECTIVE: To explore whether a brief intervention offered to frequent attenders to hospital emergency department (ED) was successful in reducing inappropriate attendance.

METHODS: Administration of the Kessler Depression and Anxiety (K10) scale; the World Health Organization Quality of Life Measure (WHOQOL-BREF); extracted de-identified rates of attendance at an ED of a general hospital and general practice, and follow up telephone interviews with study participants were used.

RESULTS: By the end of the project, a total of 53 participants had completed the programme. On average, these participants reduced their attendance at the ED significantly while demonstrating no change in their attendance rates at general practice. Additionally, they reported a decrease in psychological distress and a positive increase in their quality of life.

CONCLUSIONS: Although the number of participants in this study does not allow for robust analysis of efficacy of the program offered, it does indicate that there is merit in continuing to develop brief intervention case management models to support behaviour change programmes in hospital EDs.
\end{abstract}

KEYWORDS: frequent attenders, emergency department attendance, brief intervention

This study sits within a growing concentration of research aimed at identifying people who frequently attended the Christchurch Hospital Emergency Department (ED) in order to trial a specific intervention programme to assist them to better manage their health-seeking behaviour. Undertaking this study holds relevance for all ED funders and clinicians, and for those delivering primary health and community support services to people who are deemed to have limited health literacy or insight into managing their health needs and their relationship with health service providers. Despite a small sample size, the key findings do indicate that further study of this population of ED attenders is warranted.
It is a commonly held view that frequent inappropriate use of EDs has a significant impact on the health spend in most Western countries (Adams, 2013). The emergence of this population, who frequently utilise emergency medical service provision is not recent. As evidenced across the literature, the evolution of health provision in New Zealand, similar to that of other first world countries, has created a number of features that support the ongoing health-seeking behaviour of this population. However, this does not translate to a ready supply of solutions within the literature addressing the burden on front-line health services attributed to this population.
${ }^{1}$ West Coast District Health Board, New Zealand

${ }^{2}$ Griffith University, Australia
AOTEAROA NEW ZEALAND SOCIAL WORK 28(4), 109-115.

CORRESPONDENCE TO: Jane George jane.george@westcoastdhb. health.nz 
With such strength of anecdotal evidence pointing towards frequent attenders of EDs being a burden on health care, it would be natural to assume that much thought and research had been applied to analysing emergency health care provision and building a profile of the frequent attender population which could assist in reducing their inappropriate health-seeking behaviour. However, it would seem that those who have embarked upon research and service development have had limited success (Richardson, 2011). The published studies that have aimed to redirect people to more appropriate health care services have reported issues with recruitment leading to a lack of breadth or depth in their research. Additionally, many of the available studies focus on thematically analysing or reviewing the existing international literature (Backman, Blomqvist, Lagerlund, CarlssonHolm, \& Adami, 2008; Bieler et al., 2012; Byrne et al., 2003; Dent, Phillips, Chenhall, \& McGregor, 2003; Hunt, Weber, Showstack, Colby, \& Callaham, 2006; Phillips, Brophy, Weiland, Chenhall, \& Dent, 2006).

A variety of methods have been employed in attempts to address the health-seeking behaviours of this cluster of ED attendees in many hospitals throughout Western countries, operating varying models of health care delivery: private or state-mandated insurance, national health provision, or a combination of these. Therefore, researchers have considered whether the financial implications of primary health care could be a driver of frequent ED attendance (Fuda \& Immekus, 2006; Hunt et al., 2006; Jones \& Thornton, 2013; LaCalle \& Rabin, 2010; Mandelberg, Kuhn, \& Kohn, 2000; Morriss et al., 2012; Nelson et al., 2011; Shippee, Shippee, Hess, \& Beebe, 2014; Shumway, Boccellari, Brien, \& Okin, 2008; Thornton, Fogarty, Jones, Ragaban, \& Simpson, 2014; Weber, Showstack, Hunt, Colby, \& Callaham, 2005).

Additionally, the profile of the frequent attender has been debated across the literature. McHale et al. (2013) present an older, disadvantaged male from a non- white ethnic background, while Michelen, Martinez, Lee and Wheeler (2006) describe disproportionate use of EDs for non-acute reasons by low income, marginalised cultural groups within the urban environment of New York City. Other researchers (Kirby, Dennis, Jayasinghe, \& Harris, 2011) found no significant difference in terms of gender, culture or socio-economic status (Aigner et al., 2006). One feature that is agreed upon across the literature is that such patients overuse health care services (Gili et al., 2011). Measurement of that overuse within the ED context varies from as little as four visits per year (Kirby et al., 2011; Michelen et al., 2006) to more than a dozen (Gili et al., 2011).

Christchurch Hospital's ED experience of frequent attenders supports the profile of a population of people who genuinely believe they are unwell and in need of urgent medical attention (Bieler et al., 2012; McHale et al., 2013; Morriss et al., 2012; Rea et al., 2010; Wooden, Air, Schrader, Wieland, \& Goldney, 2009). This group are often seen by front-line staff as inappropriate attenders of ED services who could be better served by attending community agencies and primary health services (Richardson, 2011).

According to Morriss et al. (2012), across primary care as a whole the top 3\% of frequent attenders utilise $15 \%$ of all appointments. Similar findings have been reported in Switzerland by Bieler et al. (2012), and the Mt Sinai Medical Center, New York in the US where figures as high as $28 \%$ of all attendances to their ED being attributed to their frequent attenders, who make up less than $8 \%$ of the presenting population (LaCalle \& Rabin, 2010). Kennedy \& Ardagh (2004), in their study of frequent attenders at Christchurch Hospital's ED, suggest that locally frequent attenders made up only $2 \%$ of the presenting population.

A descriptive retrospective cohort study commenced in 1997 formed the basis for examining the population of frequent attenders at Christchurch Hospital (Kennedy \& Ardagh, 2004). These authors aimed to explore the idea 
that frequency of attendance was increasing in that hospital's Accident and ED (Helliwell, Hider, \& Ardagh, 2001). What they discovered, however, was that the population of patients who frequently attend is constantly changing rather than increasing in volume over time (Kennedy \& Ardagh, 2004).

\section{The Health and Wellbeing Connection pilot study}

The study reported here aimed to further explore some aspects of the success of the Health and Wellbeing Connection (HWC) pilot programme that was offered to the general adult population who frequently attended the Christchurch Hospital ED.

The overall aim of the HWC project was to strengthen primary care service providers to work with people with mild to moderate mental health needs while trialling a potential intervention service (Andrews, 2014). This programme was offered to all adults who were identified by the Christchurch Hospital's ED staff as frequent users with unmet psychosocial needs. Attendance of the pilot service was expected to result in a reduction of inappropriate ED presentations and improve overall health and wellbeing.

The first objective of the programme was to reach the target audience. The indicators of success for this objective were set as (a) psychological distress and (b) income inequality, determined by welfare benefit status. The second objective of the programme was to reduce ED attendances in a way that was attributable to the HWC programme. The third objective was to leave people better off; by meeting their immediate needs, assisting service users to better navigate health systems and assisting them to meet their goals to improve their quality of life (Andrews, 2014). The indicators of success for this objective were (a) a reduction in psychological distress, (b) improved wellbeing and (c) increased self-management.

The programme was delivered as a 12-week programme of intervention and education.
This aligned with Richmond's intentional practice framework, as the programme was delivered on a 12-week course of assessment, goal setting, partnered goal attainment, review and evaluation. Using Rapp and Goscha's strengths model (2011) as its foundation, intentional practice guides practitioners and clients through a structured programme of assessment, goal setting and supported action, framed by the key practice pillars of engagement and regular evaluation. This model, along with a commitment to providing client-centred, evidence-informed, outcomes-focussed practice was being used by Richmond at the time of this programme across the majority of their community support work services.

For the 36 participants who attended the full programme of support, there was flexibility in service frequency and length of service that was established between the HWC co-ordinator and participant. This process ensured that intervention could be tailored to the needs of the individual, and sessions could be delivered at more or less frequent intervals as the participant became more confident and skilled in navigating their health journey. Initial sessions could be scheduled every two or three days, reducing over time as the participant became more skilled at leading the process and engaged in positive and enjoyable activities relating to their goals.

\section{Method}

Mixed methods were used to evaluate the HWC programme. De-identified quantitative data was extracted from the client information systems of the ED and general practitioners involved. Levels of psychological distress were determined using scores on the Kessler 10 Depression and Anxiety Scale (K10), commonly used as a screening and outcome measurement tool recognisable within the general practice setting. In application, participants were asked to rate their frequency of certain psychological experiences, using a five-point Likert scale (Andrews \& Slade, 2001). The World Health Organization Quality of Life Measure 
(WHOQOL-BREF), used under license from Auckland University of Technology, was administered to ascertain participants' quality of life using a series of 26 questions, covering five facets of quality of life.

On completion of the programme, qualitative data were collected using semistructured questionnaires during telephone interviews with the participants and general practitioners. These interviews were conducted by an evaluator external to the programme; this evaluator was employed by Richmond, was tasked with undertaking all programme evaluation, and anonymising any data required for this study. Participants were asked to rate specific aspects of the programme and to provide oral feedback about what they valued most about the service; the impact it has had on them and any suggestions for improvement. In addition, general practitioners were asked if their expectations of the pilot were met, any key barriers and enablers experienced; what they valued most, and how the programme impacted on their service.

Data analysis was undertaken in several ways: thematic coding and analysis of the data collected in the telephone interviews $(n=17)$, a review of participant and service documentation, and thematic coding and analysis of general practitioner interviews $(n=9)$. Pegasus and the Christchurch Hospital ED provided financial data regarding the number of visits participants made, with attributed costs for each ED and GPs visit.

The design for the fieldwork for this study was built on the evaluation undertaken of the HWC programme. In order to adhere to the ethical and organisational requirements, all data utilised in this secondary analysis was anonymised by way of a numeric identifier.

\section{Results}

The characteristics of the sample consisted of 105 participants. Recruitment of the target group was achieved, as was success in each of the three objective areas. Of the participants,
$84 \%$ were experiencing psychological distress $(n=37)$ and eight out of ten participants were receiving a benefit $(n=57)$. Additionally, attendance at ED was reduced by $50 \%$. Thirteen of the 17 participants interviewed report the change was due to programme they attended. In terms of the third objective, participants were able to demonstrate that they were better off in terms of wellbeing, there was a measurable decrease in psychological distress and increase in quality of life and a reported improvement by participants in their ability to self-manage their health journey (Andrews, 2014).

Attendance rates prior to referral to the programme had a mean score of 7.48 visits in the previous 12 months across the two groups, with participants averaging just under seven visits $(6.98, \mathrm{SD}=5.390)$ while the control group were averaging one more visit each $(7.98, \mathrm{SD}=6.918)$. By the conclusion of the programme and at the point of Richmond's evaluation, which took place up to six months after intervention ceased for some participants, there was a considerable reduction in the mean number of presentations. The difference between the two groups had also widened, with participants presenting an average of 4.47 times compared to the control group with 6.67 times in the previous 12 months.

Despite a 50\% reduction in ED attendance, some participants did not demonstrate any change in their attendance rates. This was a key finding, which aligned with the literature around the subset of participants for whom circumstances such as chronic pain, lack of access to specific services such as detox or rehabilitation, or persistent homelessness makes case management less effective (Skinner, Carter, \& Haxton, 2009).

\section{Discussion}

The participants in this study clearly met the definition of frequent attender as defined by other researchers (Althaus et al., 2013; Bieler et al., 2012; Byrne et al., 2003; Hunt et al., 2006; Locker, Baston, Mason, \& Nicholl, 2007; 
Markham \& Graudins, 2011; Richardson, 2011). While meeting the criteria for this definition, the participants did not consider that they visited the ED too frequently nor had they considered that others might perceive them as doing so. However, as the participants progressed through the programme they were able to recognise that there were different options available for them to meet their health needs in the future.

The use of a client-centred goal setting approach appeared to encourage behaviour change in relation to participants making inappropriate or non-acute ED presentations to Christchurch's ED. While many participants may not have recognised this apparent effect on their behaviour, they were eager to work towards goals that mattered to them. Following the programme, they were able to reflect on how achieving those goals had offered widespread benefits, such as being too busy with new activities to worry about their health.

The participants did respond positively to developing the skills required to navigate health systems and demonstrated an ongoing ability to use these skills both within the health system and in more generalised ways. They were also able to appreciate extrinsic as well as intrinsic rewards. Simple activities such as meeting the co-ordinator at the local library for a session led to new knowledge about how to access free services and resources they had not known about previously.

The recruitment of two long-standing and highly successful staff members of Richmond's front-line staff group to the co-ordinator positions was a significant strength of this study. These two staff members were motivated individuals with a solid grasp of the practice framework delivered by Richmond and a desire to work alongside a group of people who were often marginalised and discriminated against, to empower and support them to achieve the goals that really mattered to them (Andrews, 2014). Participants attributed their success to the work that they had achieved in partnership with their co-ordinators.

\section{Ethics process}

Retrospective ethics approval was sought for the study from the University of Otago's Ethics Committee, who deemed that ethical approval for this study was not required as only unidentified data would be used, thereby meeting the Health Information Privacy Code Rule 11 (2) (c) (ii) (Health Information Privacy Code, 1994).

\section{Limitations of study}

A significant limitation of our study was the inability to access some data towards the end of the study due to the change of personnel in each of the organisations. Being able to ensure that organisational commitment to the sharing of data is more robust may be challenging for researchers, but it is worthy of consideration in terms of the drawing up of a memorandum of understanding should a dual research/business analysis process to facilitate in the future.

Further analysis of the specific participants and the control group of this study relating to their ongoing relationship with the ED would offer further insight into whether the learnings gained through a case management approach such as this, which teaches independence in health system navigation, can be sustained, or whether a reduction in attendance is actually just a natural ebb and flow (Skinner et al., 2009) as some literature suggests.

For this study, and for the HWC project another limitation was the low numbers of participants who were willing to participate in the programme, which was coupled with the reluctance of ED staff to complete any extra paperwork to refer potential participants to the programme. Developing a more robust and comprehensive recruitment strategy prior to launching future programmes would allow for the potential of a larger sample size and more people to benefit from participation in the programme (Andrews, 2014). 
Alongside this was the lack of IT connectivity or infrastructure to allow real-time identification of participants, electronic referral to the programme from ED or sharing of case records between the HWC and the ED clinicians. This limitation was also a contributing factor in recruitment (George, Cole, \& Andrews, 2013).

Consideration should also be given to other programmes being developed, trialled or delivered within the ED environment for similar populations when developing a programme such as this. Where competing programmes exist, there is a risk of limiting the potential participant population or tainting the results should participants inadvertently participate in more than one programme.

\section{Conclusion}

Emergency departments are chaotic, noisy and adrenaline fuelled places. They attract a staff group who love a fast paced, anything can happen workplace. They also attract a group of the community, who visit regularly for reasons that do not seem, to the staff at least, to fit the nature of ED business.

This group, though small in number, make up a significant proportion of the visits recorded in each 24-hour period. Study participants, described as frequent flyers or worried well, are defined as those who visit the ED when their needs could be met in general practice, and who visit the ED more than four times each year.

Understanding how people shape their view of who is responsible for their health is a crucial to any process of health-related behaviour change. For some people, their view of health is concrete: Their GP is responsible for keeping them well and healthy. For others, the entire responsibility for their health depends on the situation, albeit at work, sport, home or in public spaces and it is also dependent on the context of age or life stage. Although the small sample size in this study does not allow for robust analysis of efficacy of an intervention it does indicate that there is merit in continuing to develop brief intervention case management models to support behaviour change programmes in EDs.

Future studies need to consider a component of analysis for each participant before any co-ordination or intervention could be proposed, as participants need to be able to acknowledge and understand their own view of health and their responsibility in order to set goals to improve their own health and wellbeing.

\section{References}

Adams, J. G. (2013). Emergency department overuse perceptions and solutions: Perceptions of ED overuse. Journal of the American Medical Association, 309(11), 2.

Aigner, M., Förster-Streffleur, S., Prause, W., Freidl, M., Weiss, M., \& Bach, M. (2006). What does the WHOQOL-BREF measure? Measurement overlap between quality of life and depressive symptomatology in chronic somatoform pain disorder. Social Psychiatry and Psychiatric Epidemiology, 41(1), 81-86. doi:10.1007/s00127-005-0997-8

Althaus, F., Stucki, S., Guyot, S., Trueb, L., Moschetti, K., Daeppen, J., \& Bodenmann, P. (2013). Characteristics of highly frequent users of a Swiss academic emergency department: A retrospective consecutive case series. European Journal of Emergency Medicine, 20(6), 413-419. doi:10.1097/MEJ.0b013e32835e078e

Andrews, S. (2014). Evaluation of the Health \& Wellbeing Connection pilot, May 2012-October 2013. Christchurch, New Zealand: Richmond Services.

Andrews, G., \& Slade, T. (2001). Interpreting scores of the Kessler Psychological Distress Scale (K10). Australian and New Zealand Journal of Public Health, 25, 494-497.

Backman, A., Blomqvist, P., Lagerlund, M., Carlsson-Holm, E., \& Adami, J. (2008). Characteristics of nonurgent patients: Cross-sectional study of emergency department and primary care patients. Scandinavian Journal of Primary Health Care, 26(3), 181-187. doi:10.1080/02813430802095838

Bieler, G., Paroz, S., Faouzi, M., Trueb, L., Vaucher, P., Althaus, F., ...Bodenmann, P. (2012). Social and medical vulnerability factors of emergency department frequent users in a universal health insurance system. Academic Emergency Medicine, 19(1), 63-68. doi:10.1111/j.1553-2712.2011.01246.x

Byrne, M., Murphy, A. W., Plunkett, P. K., McGee, H. M., Murray, A., \& Bury, G. (2003). Frequent attenders to an emergency department: A study of primary health care use, medical profile, and psychosocial characteristics. Annals of Emergency Medicine, 41(3), 309-318. doi:10.1067/mem.2003.68

Dent, A. W., Phillips, G. A., Chenhall, A. J., \& McGregor, L. R. (2003). The heaviest repeat users of an inner city 
emergency department are not general practice patients. Emergency Medicine, 15(4), 322-329. doi:10.1046/ j.1442-2026.2003.00470.x

Fuda, K. K., \& Immekus, R. (2006). Frequent users of Massachusetts emergency departments: A statewide analysis. Annals of Emergency Medicine, 48(1), 9-16.

George, J., Cole, M., \& Andrews, S. (2013). The Health \& Wellbeing Connection: An integrated assessment, co-ordination \& navigation pilot service within a primary health setting. Christchurch, New Zealand: Richmond New Zealand Ltd.

Gili, M., Luciano, J. V., Serrano, M. J., Jimenez, R., Bauza, N., \& Roca, M. (2011). Mental disorders among frequent attenders in primary care: A comparison with routine attenders. Journal of Nervous and Mental Disease, 199(10), 744-749. doi:10.1097/NMD.0b013e31822fcd4d

Health Information Privacy Code. (1994). Part 6 of the Privacy Act, No. 28 (1993).

Helliwell, P., Hider, P., \& Ardagh, M. (2001). Frequent attenders at Christchurch Hospital's emergency department. New Zealand Medical Journal, 114(1129), 160-161.

Hunt, K., Weber, E., Showstack, J., Colby, D. C., \& Callaham, M. (2006). Characteristics of frequent users of emergency departments. Annals of Emergency Medicine, 48(1), 1-8. doi:10.1016/j.annemergmed.2005.12.030

Jones, P. G., \& Thornton, V. (2013). Does cost drive primary care patients to New Zealand's emergency departments? A systematic review. New Zealand Medical Journal, 126(1387), 15

Kennedy, D., \& Ardagh, M. (2004). Frequent attenders at Christchurch Hospital's emergency department: A 4-year study of attendance patterns. New Zealand Medical Journal Online, 117(1193).

Kirby, S. E., Dennis, S. M., Jayasinghe, U. W., \& Harris, M. F. (2011). Frequent emergency attenders: Is there a better way? Australian Health Review, 35(4), 462-467. doi:10.1071/AH10964

LaCalle, E., \& Rabin, E. (2010). Frequent users of emergency departments: The myths, the data, and the policy implications. Annals of Emergency Medicine, 56(1), 42-48. doi:10.1016/j.annemergmed.2010.01.032

Locker, T., Baston, S., Mason, S. M., \& Nicholl, J. (2007). Defining frequent use of an urban emergency department. Emergency Medicine Journal, 24(6), 398-401. doi:10.1136/emj.2006.043844

Mandelberg, J. H., Kuhn, R. E., \& Kohn, M. A. (2000). Epidemiologic analysis of an urban, public emergency department's frequent users. Academic Emergency Medicine, 7(6), 637-646. doi:10.1111/j.1553-2712.2000.tb02037.x

Markham, D., \& Graudins, A. (2011). Characteristics of frequent emergency department presenters to an Australian emergency medicine network. BMC Emergency Medicine, 11, (1) 21. doi:10.1186/ 1471-227X-11-21

McHale, P. W., Wood, S., Hughes, K., Bellis, M. A. Demnitz, U., \& Wyke, S. (2013). Who uses emergency departments inappropriately and when. BMC Medicine, 11(2013), 258

Michelen, W. M., Martinez, J., Lee, A., \& Wheeler, D. P. (2006). Reducing frequent flyer emergency department visits. Journal of Health Care for the Poor and Undeserved, 17(1), 59-69.
Morriss, R., Kai, J., Atha, C., Avery, A., Bayes, S., Franklin, M., ...Yang, Min. (2012). Persistent frequent attenders in primary care: Costs, reasons for attendance, organisation of care and potential for cognitive behavioural therapeutic intervention. BMC Family Practice, 13(39). doi:10.1186/1471-2296-13-39

Nelson, K., Connor, M., Wensley, C., Moss, C., Pack, M., \& Hussey, T. (2011). People who present on multiple occasions to emergency departments. Emergency Medicine Australasia, 23, (5)532-540.

Phillips, G., Brophy, D., Weiland, T., Chenhall, A., \& Dent, A. (2006). The effect of multidisciplinary case management on selected outcomes for frequent attenders at an emergency department. Medical Journal of Australia, 184(12), 602-606. Privacy Act, No. 28 (1993). Retrieved from http://www.legislation.govt.nz/act/public/1993/0028/ latest/DLM296639.html (accessed 29.03.2015).

Rapp, C. A. \& Goscha. R. J. (2011). The strengths model: A recovery-oriented approach to mental health services, 3rd ed. Oxford, United Kingdom: Oxford University Press.

Rea, H., Kenealy, T., Horwood, F., Sheridan, N., Parsons, M., Wemekamp, B., ...Degelong, P. (2010). Integrated systems to improve care for very high intensity users of hospital emergency department and for long-term conditions in the community. New Zealand Medical Journal Online, 123(1320), 76-85.

Richardson, S. K. (2011). Defining appropriateness of emergency department attendance: $A N Z$ perspective (Unpublished doctoral thesis). University of Otago, Dunedin, New Zealand.

Shippee, N., Shippee, T. P., Hess, E. P., \& Beebe, T. J. (2014). An observational study of emergency department utilization among enrollees of Minnesota Health Care Programs: Financial and non-financial barriers have different associations. BMC Health Services Research, 14 (1) NP. doi:10.1186/1472-6963-14-62

Shumway, M., Boccellari, A., Brien, K., \& Okin, R. (2008). Cost-effectiveness of clinical case management for ED frequent users: Results of a randomized trial. American Journal of Emergency Medicine, 26(2), 155-164. doi:10.1016/j.ajem.2007.04.021

Skinner, J., Carter, L., \& Haxton, C. (2009). Case management of patients who frequently present to a Scottish emergency department. Emergency Medicine Journal, 26(2), 103-105. doi:10.1136/ emj.2008.063081

Thornton, V., Fogarty, A., Jones, P., Ragaban, N., \& Simpson, C. (2014). Why do patients self-present to Middlemore Hospital Emergency Department? New Zealand Medical Journal, 127(1394), 19-30.

Weber, E. J., Showstack, J. A., Hunt, K. A., Colby, D. C., \& Callaham, M. L. (2005). Does lack of a usual source of care or health insurance increase the likelihood of an emergency department visit? Results of a national population-based study. Annals of Emergency Medicine, 45(1), 4-12. doi:10.1016/j. annemergmed.2004.06.023

Wooden, M. D., Air, T. M., Schrader, G. D., Wieland, B., \& Goldney, R. D. (2009). Frequent attenders with mental disorders at a general hospital emergency department. Emergency Medicine Australasia, 21(3), 191-195. doi:10.1111/j.1742-6723.2009.01181.x 